\title{
Implementation of Advertising Machine Based on The Intelligent Terminal
}

\author{
Zhiyuan Zhang, Xiangdong You, Zhixin Zhang and Fan Li \\ Beijing University of Posts and Telecommunications, Beijing 100876, China \\ zhangzhiyuan@bupt.edu.cn, youxiangdong@bupt.edu.cn, uuytap@163.com, lifan@bupt.edu.cn
}

Keywords: Network Advertising; Android; MiniPC

\begin{abstract}
With the rapid development of China's economy, advertising has already become the main means of businesses, enterprises and departments to promote their advertising. Using advertising to publish advertising has gradually been widely recognized and accepted by all people. In this context, through the use of efficient players and network technology, advertising content updates and remote control playback is meaningful. Multimedia network advertising system with its low cost, high stability, good real-time performance, ease of management, etc., showing good application and development prospects. This paper summarizes and analyses the results of previous studies, combine the advanced Android system and effective advertising, design and implement a kind of advanced network advertising system with the core of Android operating system.
\end{abstract}

\section{Introduction}

As is known to all, the emergence of advertising is the necessity of developing business. Good advertisement can bring good opportunity, promote the prosperity of business. Both are mutually beneficial coexistence. In recent years, with the rapid development of China's economy and urban construction, the increase of the number of travelling and leisure activity, made the advertising industry enter into a new stage [1].

Online advertising machine realizes the information display, video advertising play and makes the convenient which allows people to control all the terminals all over the world at home and it extremely decrease the labor costs, enhance the efficiency, and follow the trend of the network.

In addition, the development of mobile communication technology also supports the development of online advertising machine. On the aspect of multimedia advertising machine system, we can also take its unique advantages to develop multimedia advertising machine system based on $3 \mathrm{G}$ network. It must satisfy the trend of the multimedia advertising machine development in the future.

\section{The requirement analysis and system design}

The requirement analysis and system design. The demand for the advertising machine system can be summarized in three points. First, the playing capacity of the advertising machine terminal needs to be customized, including the content of the advertising media, the playing order of the advertising media and the playing time of the advertising media. Second, all the terminals must be managed by the centralized control server. Third, the network connection must be di-versify, at least supports wired Ethernet connection, WIFI connection and 3G connection.

(1) Customized playing capacity of advertising machine terminal

Advertising machine terminal's playing capacity can be customized including content and patterns. Customized content refers the advertising content can be updated through the network; Customized patterns refers to the media on advertising machine which is controlled by playlists, and the playlists can be updated via the Internet.

(2) Centralized management of the control server

Centralized management of the control server refers that the system administrator can customized manage all the advertising machine terminals through the network.

(3) Diversity of network connection 
The diversity of the network connection refers that the advertising machine terminal can access system not only through the cable Internet, but also via WIFI or 3G network, and measures must be took for net-work anomalies, such as network outages and non-stable network.

In summary, the application scenarios of the sys-tem is that when the advertising machine terminal access to the system via Internet, the administrators can manage the centralized and grouped advertising ma-chine system only if they log into the central control server.

The whole architecture of the system. Considering the above analysis of the demand, the development cycle and performance effect, the overall structure design of the system can be divided into three parts, including embedded advertising machine terminal, centralized control server and network connection.

Embedded terminal advertising machine. Advertising machine terminals mainly receive multimedia advertising data distributed from centralized control server, and then display the data. According to the demand analysis, advertising machine terminal should have ability to play audios, video files, pictures, and connect the centralized control server via network.

Based on the analysis of the above, we put forward the advertising machine design scheme, which the hardware is based on the intelligent terminal, and the software is based on the android operating system. Figure 1 shows the software structure.

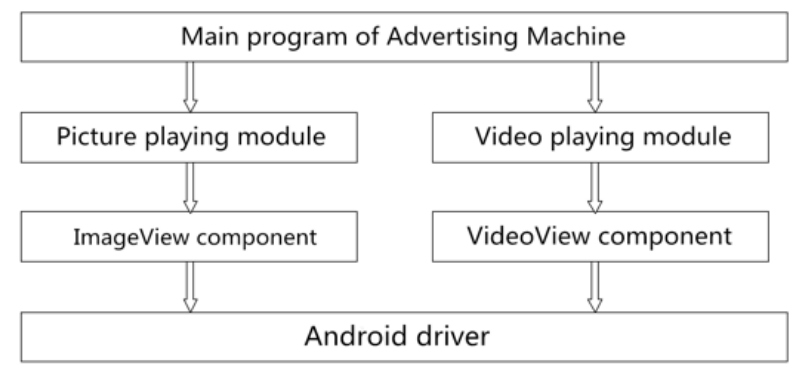

Figure 1. The software structure.

The main idea of the program is to show the picture and video according to the playlist. Call the module of image-playing via the ImageView component to implement when the picture is detected, and call the module of video-playing via the VideoView component to implement when the video is detected.

Centralized control server. According to actual requirements, the network environment of the centralized control server can be either in the LAN which is the same as the environment of the advertising machine terminal or the wide network. And the latter one is more general.

Centralized control server mainly manages numerous advertising machine terminals with centralized management and group management, and stores the multimedia advertising data resources.

The system supports the traditional wired Ethernet connection, in order to make the advertisement machine terminal flexible and convenient. And it also support WIFI and 3G mobile connections, as long as a hotspot is in the area, or 3G network covers the area, so it can be placed anywhere at any time, and can be convenient and efficient.

The design of the network connection. For advertising machine system control mode, there are two main types to choose, Pull mode and Push mode. The two models have their advantages and dis-advantages [2].

(1) Pull mode

The advantage of the Pull mode is the centralized control terminal server and the network advertising machine can be put in different position which is in different network, as long as the centralized control server monitor is in the network which is available to the outside. While the disadvantage of the Pull model is that the model wastes network bandwidth to some extent because the network advertising machine terminal need to periodically send query data to the central control server. 


\section{(2) Push model}

The Push model refers to the embedded terminal advertising machine in the listening state, the centralized control server send the media data to the advertisement machine terminal via the network and then play them when there is updated.

The advantage of Push mode is that the advertisement machine terminal does not need to waste additional network bandwidth due to the fact that the centralized control server actively push the media data to network advertising machine terminal. And the disadvantage of the Pull mode is that the advertising ma-chine terminal must reach the centralized control server because the advertising machine terminals are al-ways in the listening state. Due to the numerous of the terminal, the centralized server and the terminals must in the same local area network (LAN) on the network topology.

Because the centralized control server can be not only in the same local area network(LAN) with the advertising terminal, but also in different network environment in the process of practical use and fully considering the decoupling between terminal and server, advertising machine only need to establish connection with the server through the server file query function indirectly. In addition, considering the changes of the network environment in the future as well as the expansion of the system across the net-work, we finally choose the pull mode as the control model of the whole system.

\section{The realization of the advertising machine terminal}

Whole Framework. As shown in Figure 2 below, we know the running process of the advertising machine. If the configuration is set before the advertisement machine connects to the server, the running process is as follow:

First thing is to check the local data file: If the data file exists, download the timestamp from the server, and then contrast the local timestamp with the down-load one. If they are same, the terminal continues to play the local file, or downloads new resources and time stamp. If there is no data file, the terminal down-loads resources and timestamp data from the server. Then it plays repeatedly according to the local list, and gets a new timestamp from the server when completing every cycle.

The above figure is a single advertisement machine terminal flow chart. In fact, the advertising machines are on a large scale. So in order to distinguish different terminals and make the centralized management of multiple advertising machine, we must give each advertising machine a single logo which is used to publish playlist or other operations.

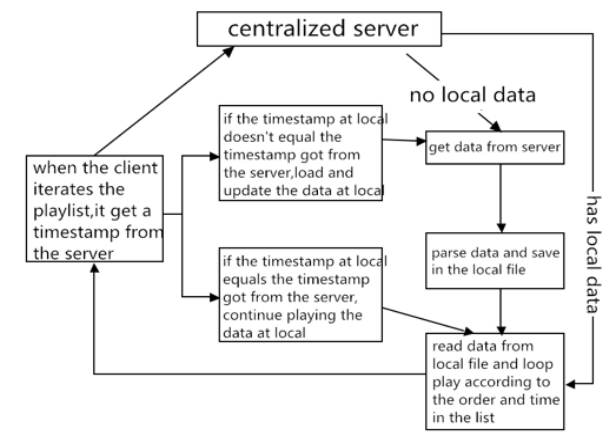

Figure 2. The running process of the system.

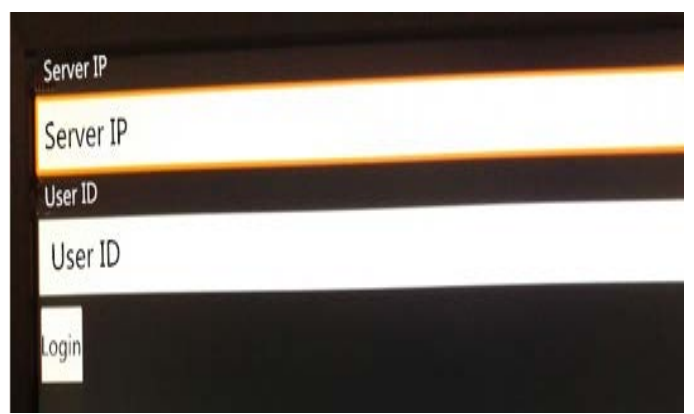

Figure 3. The login interface.

As shown in Figure 3, the user can login into the network advertising machine with the terminal server url and user id, so that the server can make the operation on the advertising machine. For example, I enter the server IP address and 01 of user id, so that the server can generate a json file called playlist_01 and it can control the playing of the advertising machine through modifying the file on the server.

Program flow. The flow chart of the program is shown in Figure 4. Advertising machine terminal should have abilities of playing the pictures, audio, video file and connecting to the centralized control server. 
Image playback function depends on ImageView components. Android provides a VideoView component which is used to play video files. Using its setVideoPath() method or setVideoURI() method to play video, and finally call the start() method to play video. The HttpURLConnection class is mainly used to connect the network[3,4].

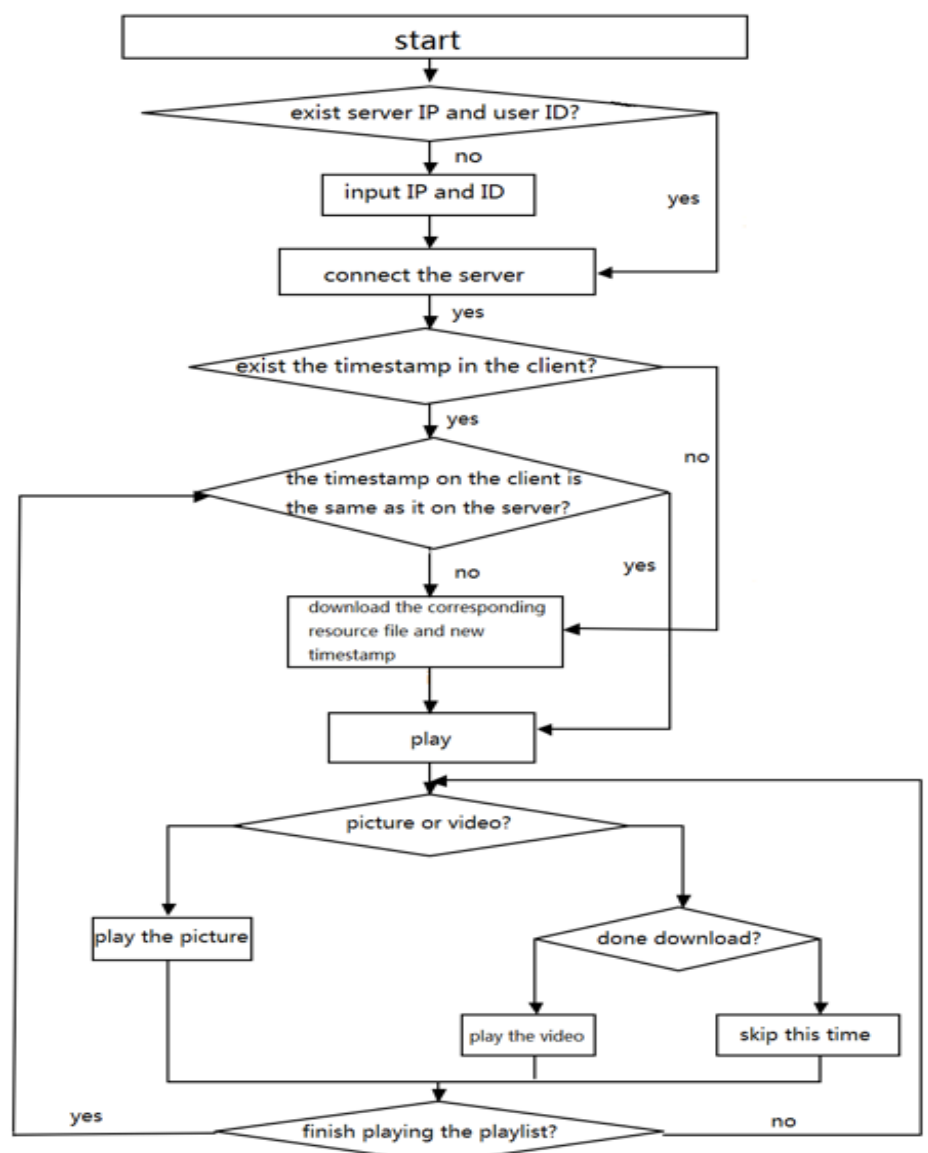

Figure 4. The running process of the client.

\section{Conclusions}

Finally, we take a completely test of the network advertising machine system. The content of testing is mainly about functional testing and performance testing.

Environment test. Environment test including: miniPC, Konka monitor, TPLINK wireless routers, a PC machine and three net-lines. Among them, two advertising machines connect to the local area network (LAN) via a cable; others connect to the LAN via WIFI.

The test case. The following test aims at testing the function of advertising machine system and the performance of Android advertising machine terminal.

Function test. Functional testing is primarily to test whether the playing model and switch model correct, and test whether the terminal can be automatically restored connection with the server after network interruption. The function of the test is as shown in Table 1. The design sketch of the software testing is as shown in Figure 5.

The performance test. Performance testing is primarily to test the performance of the terminal and the system in the long running time.

When choosing the developer options, we can click the item to show the CPU usage rate. There will be some data shown in the following Figure 6.

Analysis. Through the test case above, we can see that the sys-tem has successfully realized the requirements in the aspects of the customized, diversity advertisement content and center control server [5].

The three numbers in Figure 6 present CPU load of the Linux/Android system, and they represent 
the estimated average load during three different intervals ( 1 minute, 5 minutes and 15 minutes).

In this case we can see that the three numbers $(1.08,1.0$ and 1.02) are quite small. They indicate that the system occupies little resource and the load of the terminal is quite little. The difference among the three numbers is little which indicates the load over a short period of time (1 minute) and a long period of time (5 minutes and 10 minutes) are similar. It also proves that the stability of the system over a long period.

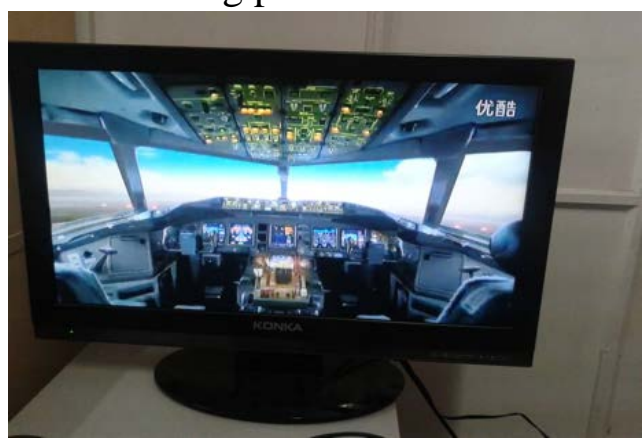

Figure 5. The screenshot of the test.

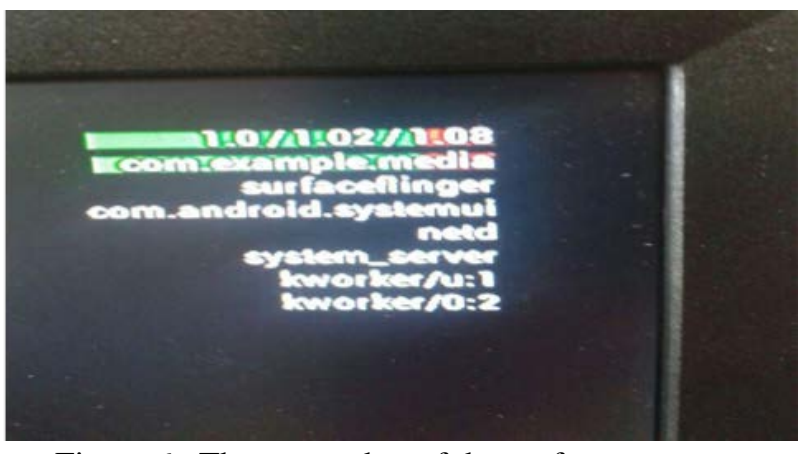

Figure 6. The screenshot of the performance test.

Table 1. The test case.

\begin{tabular}{|c|l|l|l|}
\hline Test no. & Test case & Procedure description & Expect result \\
\hline 1 & Test picture & $\begin{array}{l}\text { Add a picture to the list on the } \\
\text { server. } \\
\text { Submit. }\end{array}$ & $\begin{array}{l}\text { Show the new picture in the } \\
\text { next time. }\end{array}$ \\
\hline 2 & Test video & $\begin{array}{l}\text { Add a short video to the list on } \\
\text { the server. } \\
\text { Submit. }\end{array}$ & $\begin{array}{l}\text { Show the new short video in the } \\
\text { next time. }\end{array}$ \\
\hline 3 & $\begin{array}{l}\text { Test the skipping function when } \\
\text { the process of downloading the } \\
\text { video has not done. }\end{array}$ & $\begin{array}{l}\text { Add a long video to the list on } \\
\text { the server. } \\
\text { Submit. }\end{array}$ & $\begin{array}{l}\text { Show the new long video after a } \\
\text { long time. }\end{array}$ \\
\hline 4 & Test the network exception & $\begin{array}{l}\text { Disconnect the network and then } \\
\text { connect it after 5 minutes. } \\
\text { Change the playlist and submit. }\end{array}$ & $\begin{array}{l}\text { Play the local file when the } \\
\text { client disconnect the network. } \\
\text { Update the list when the } \\
\text { connection is established again. }\end{array}$ \\
\hline
\end{tabular}

Conclusion. The system adopts the miniPC and Konka monitor as the hardware platform of Android advertising ma-chine terminal. Take advantage of its processing capacity of video, image and other multimedia data to improve performance of playback and user experience. The system also implements the network advertising machine system, and analyzes the system performance. The analysis shows that the system can completely satisfy the requirements listed at the be-ginning.

In addition, the paper describes that the network advertising machine system is innovative in many aspects such as supporting flexible network access, centralized management of the system, customized mode and contents of the advertisements.

\section{References}

[1] Yeming Fan: Design and implementation of network advertising machine. Dalian Maritime University. 2009.

[2] Information on http://www.cnblogs.com/hanyonglu/archive/2012/03/04/2378971.html

[3] Mingyu Yang: Android grammar example references (Electronic Industry Press, 2012). In Chinese.

[4] Ning Li: Android development authority guide (People's posts and telecommunications Press, 2011). In Chinese.

[5] Information on http://blog.chinaunix.net/uid-13153795-id- 2906401.html 